\title{
Time scale similarity and long-term memory for autobiographical events
}

\author{
BRYAN J. Moreton AND GeOFF WARD \\ University of Essex, Colchester, England
}

\begin{abstract}
We examine the extent to which retrieval from very long-term autobiographical memory is similar when participants are asked to retrieve from widely differing periods of time. Three groups of 20 participants were given 4 min to recall autobiographical events from the last 5 weeks, 5 months, or 5 years. Following recall, the participants dated their events. Similar retrieval rates, relative recency effects, and relative lag-recency effects were found, despite the fact that the considered time scales varied by a factor of 52 . These data are broadly consistent with the principle of recency, the principle of contiguity (Howard \& Kahana, 2002), and scale similarity in the rates of recall (Brown, Neath, \& Chater, 2007; Maylor, Chater, \& Brown, 2001). These findings are taken as support for models of memory that predict time scale similarity in retrieval, such as SIMPLE (Brown et al., 2007) and TCM (Howard \& Kahana, 2002).
\end{abstract}

It has recently been claimed that a characteristic of human memory is its tendency to show similar performance on tests across different time scales (e.g., Brown, Neath, \& Chater, 2007; Howard \& Kahana, 2002; Maylor, Chater, \& Brown, 2001), a property that is known as time scale similarity. These time scale similarities are central to recent theories that view episodic memory as a continuum, such as the temporal context model (TCM; Howard \& Kahana, 2002) and the scale-independent memory, perception, and learning model (SIMPLE; Brown et al., 2007).

For example, time scale similarities in retrieval rates have been claimed on the basis of the observation that rates of recall from autobiographical memory may be highly similar over widely differing time periods (Maylor et al., 2001). Time scale similarity is also assumed in the principle of recency (Howard \& Kahana, 2002), which encapsulates the commonly obtained recall advantage for more recently encountered events. Indeed, recency effects have been observed over seconds (Murdock, 1962), weeks, and years (Rubin, 1982). Moreover, the principle of contiguity (Howard \& Kahana, 2002) refers to the observed regularities in the output order in free recall, where there is a recall advantage for items that were studied in close temporal proximity to those that have just been retrieved (Howard \& Kahana, 1999), and this has been observed over seconds and also minutes (Howard, Youker, \& Venkatadass, 2008; Unsworth, 2008).

The main aim of this research was to provide quantitative tests of time scale similarity in all three retrieval measures in a single experiment that simultaneously examined retrieval over time periods far greater than those previously covered by the principle of contiguity. In so doing, we replicated and extended an experiment that Maylor et al. (2001) conducted to examine time scale similarities in retrieval from autobiographical memories.

Before detailing the Maylor et al. (2001) study, we should first acknowledge that one loses some aspects of experimental control when one asks participants to retrieve autobiographical memories. In laboratory list-learning experiments, the to-be-remembered items are randomly selected from an experimental pool of stimuli and are randomly ordered. By contrast, participants' autobiographical memories are idiosyncratic to each individual and are inherently organized, such that time is confounded with other, nontemporal kinds of organization of life events that can shape retrieval. We will return to this point in our discussion.

Maylor et al. (2001) asked three groups of participants to recall jobs, appointments, and other things that they had done from one of three time periods: from the last day, the last week, or the last year. The total recall time was 4 min, which was subdivided into eight recall periods of $30 \mathrm{sec}$. During the first 30 -sec recall period, the participants wrote down their events in the first section of their response sheet. However, after every 30 -sec recall period, they were asked to finish the event they were currently recording and then move to the next section of their response sheet. Maylor et al. found that the participants' retrieval rates and totals were virtually indistinguishable: The participants recalled a total of about 20 items in each of the three time scales, starting with approximately 5 items in Recall Period 1 and ending with 1 item in Recall Period 8. Maylor et al. also found similar retrieval rates for the retrieval of events planned for the next day, the next week, or the next year. Penningroth (2005) has since

B. J. Moreton, bryan.moreton@nottingham.ac.uk 
replicated the three past and future conditions and found similar results.

Although the results reported by Maylor et al. (2001) are consistent with time scale similarity, the invariance in the total number of items retrieved from very different time periods seems contrary to the list length effects observed in laboratory list-learning experiments (e.g., Murdock, 1962; Ward, 2002), in which participants tend to recall more items, on average, from longer lists (albeit at a lower proportion). For example, Ward asked participants to rehearse out loud while learning lists of 10,20 , or 30 words. He found that more words were recalled from longer lists and that recall was affected by the recency of the most recent rehearsal of these list items. Indeed, the recency-based retention functions for each list length could be superimposed upon one another.

More generally, the data from Ward (2002) highlight a potential source of tension. If accounts of list length effects are related to recency, how can the principle of recency be accommodated simultaneously with timescale-similar retrieval rates? One might reasonably expect that the accessibility of to-be-remembered events would decrease with increasing retention interval (recency effects). However, whereas Ward predicted that more events should be recalled from the last year than from the last day, because all of the events in the latter time scale are included in the former time scale, the data from Maylor et al. (2001) showed that recall is largely invariant across largely varying time periods. Unfortunately, it is not possible to see whether or how the time scale similarities in recency and retrieval rates co-occur in Maylor et al.'s data, because the retention functions of the different conditions were not reported.

Therefore, we asked three groups of 20 participants to recall autobiographical events from the last 5 weeks, 5 months, or 5 years. Following recall, we asked the participants to date their memories, such that the scale-similar properties of rates of recall, recency, and contiguity could be examined simultaneously across the very different time scales.

\section{METHOD}

\section{Participants}

Sixty-three undergraduate students from the University of Essex were given course credit in return for participation.

\section{Materials and Apparatus \\ The experiment and instructions were presented using the Super- Card 4.5 application, via an Apple Macintosh computer. The par- ticipants also received response sheets and blank calendars. The response sheets were divided into eight numbered sections (double sided; Sections 1-4 on Side 1, Sections 5-8 on Side 2) correspond- ing to the eight recall periods of $30 \mathrm{sec}$. Each section of the response sheet had 20 vertically aligned bullet points. The blank calendars showed the dates from January 2003 to the month of testing (Octo- ber or November 2008), and each participant had the date that he or she was tested clearly marked.}

\section{Design}

The experiment used a $3 \times 8$ mixed design. The between-subjects factor was the temporal condition, with three levels ( 5 weeks, 5 months, and 5 years). The within-subjects factor was the recall pe- riod, with eight levels (Recall Periods 1-8). The dependent variable was the number of autobiographical events retrieved.

\section{Procedure}

The participants were assigned to one of the three temporal conditions, according to their order of arrival. They were instructed to recall as many autobiographical events as possible from the last 5 weeks, 5 months, or 5 years. They were asked to summarize their events into one or two words and to record these summaries on their response sheets. The total response time was $4 \mathrm{~min}$, which was subdivided into eight recall periods of $30 \mathrm{sec}$ each. During the first recall period, the participants recorded their events in the first section of their response sheet, starting at the top bullet point and moving down to the next bullet point after every consecutively recalled event. A tone at the end of each 30 -sec recall period signaled that the participant should move to the next section of the response sheet. After the last recall period, the participants were told to return to their autobiographical events and provide a date of occurrence estimate for each event. The participants were instructed to be as accurate as possible (i.e., day, month, and year), but if they were unsure of the date of occurrence, they were asked to make an educated guess. The participants were permitted to use the calendars provided during the task in order to aid their date estimates, and they were given a maximum of $5 \mathrm{~min}$ to complete the dating procedure.

\section{RESULTS}

Three participants were rejected because they failed to follow task instructions, leaving 20 participants in each group, who retrieved a total of 1,223 autobiographical events.

\section{Retrieval Rates}

Figure 1 shows the mean number of autobiographical events retrieved for each of the three temporal conditions across the eight recall periods. A 3 (temporal condition) $\times$ 8 (recall period) mixed design ANOVA was conducted to compare the retrieve rates for the three conditions. There was a nonsignificant main effect of temporal condition $[F(2,57)<1]$. There was a highly significant main effect of recall period $\left[F(7,399)=30.62, M S_{\mathrm{e}}=1.06\right.$, $\left.p<.001, \eta_{\mathrm{p}}^{2}=.35\right]$, and a nonsignificant interaction $[F(14,399)=1]$. Tukey HSD tests indicated that the number of recalled events decreased across the recall periods $(p s<.05)$.

\section{Participant-Dated Retention Functions}

The retention functions were calculated on the basis of the date estimates that were provided by the participants. The participants did not always provide an exact date, and so some date estimates were converted into exact dates by applying the following rules: (1) If the participants provided a range of days, the middle of the range was used, and (2) if the participants indicated that the event had occurred at the beginning or end of a particular month, the first or last day, respectively, was used. Of the 1,223 recalled autobiographical events, 93 (8\%) could not be date estimated.

Figure 2A shows the absolute retention functions of the recalled events, in which retention is measured in weeks between the date estimates and the day of test. The figure shows that a greater number of recent events than of distant events were recalled, demonstrating recency, but the retention functions of the three groups do not superimpose 


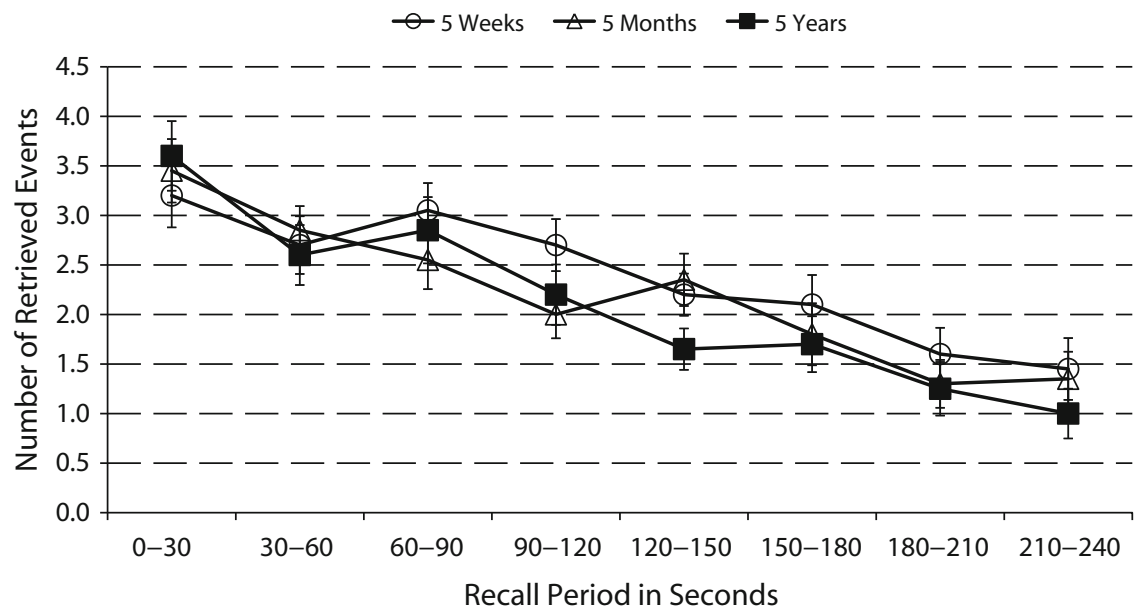

Figure 1. Mean number of autobiographical events retrieved in each of the three temporal conditions and eight recall periods. Error bars represent \pm 1 standard error.

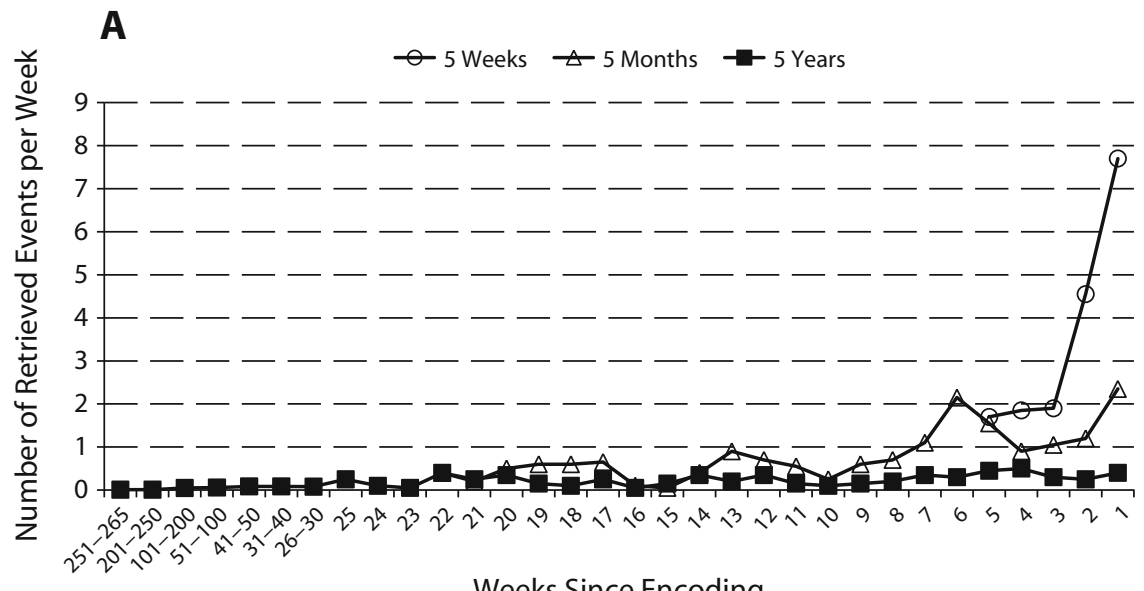

Weeks Since Encoding

B

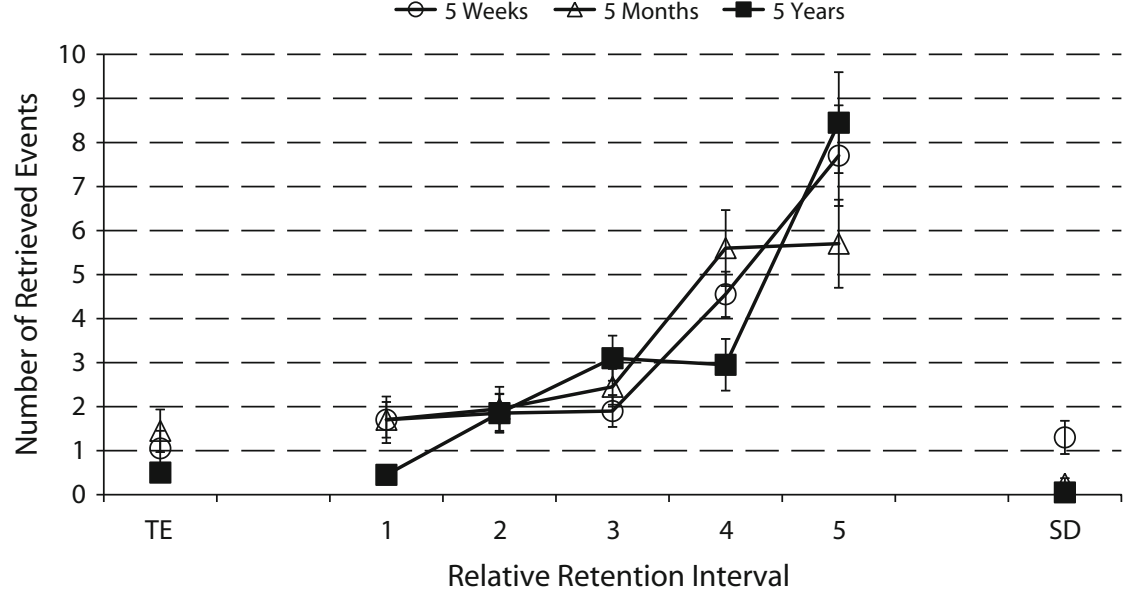

Figure 2. Retention functions for the recalled autobiographical events. (A) Mean number of autobiographical events retrieved per week since encoding. (B) Mean number of autobiographical events retrieved at each relative retention interval (RRI). High RRIs refer to more recent events, and low RRIs refer to less recent events, within each temporal condition. Temporal errors (TEs) refer to date estimates that exceeded the temporal period. Same day (SD) events represent date estimates that referred to the day of test. Error bars represent \pm 1 standard error. 
upon one another: The participants in the 5-weeks condition recalled more events from the last 5 weeks than did those in the 5-months condition $[t(38)=5.94, p<.001]$, and the participants in the 5-months condition recalled more events from the last 5 months than did those in the 5 -years condition $[t(38)=7.20, p<.001]$. It is clear from Figure 2A that the number of memories retrieved from any given time period (e.g., the most recent week) is rather different, depending on the time scale over which participants search their memories.

However, the same absolute time period (e.g., the most recent week) reflects different proportions of the three different scales. In order to examine the retention functions using intervals that were scaled so that they constituted equivalent proportions of each total temporal period, the date estimates were categorized into one of five relative retention intervals (RRIs). Each RRI corresponded to a fifth of each time scale, such that 1 RRI corresponded to 1 week in the 5-weeks condition, 1 month in the 5-months condition, and 1 year in the 5 -years condition.

Figure 2B shows the mean number of events that were retrieved from each of the five RRIs for the three temporal conditions. In contrast to Figure 2A, Figure 2B shows rather similar retention functions when the same data are rescaled as a proportion of the total temporal period. In Figure 2B, RRI 5 refers to the most recent interval, whereas RRI 1 represents the least recent interval. These data were analyzed using a 3 (temporal condition) $\times$ 5 (RRI) mixed design ANOVA. There was no significant main effect of temporal condition $[F(2,57)<1]$, a highly significant main effect of RRI $\left[F(4,228)=38.92, M S_{\mathrm{e}}=\right.$ $\left.9.10, p<.001, \eta_{\mathrm{p}}^{2}=.41\right]$, and a significant interaction $\left[F(8,228)=2.55, M S_{\mathrm{e}}=9.10, p<.05, \eta_{\mathrm{p}}^{2}=.08\right]$. An analysis of the simple main effects revealed that all three temporal conditions showed highly significant relative recency effects $(p s<.001)$ but that the temporal conditions were significantly different at RRIs 4 and 5. Unpaired $t$ tests indicated that the 5 -months condition produced slightly fewer events than did the other two conditions at RRI $5(p s<.05)$ but slightly more events than did the 5 -years condition at RRI $4(p<.01)$. We note that for the 5 -months condition, RRI 4 fell at the end of the summer holidays and the beginning of the academic year for the student participants and could, therefore, have been due to a calendar type effect (see, e.g., Anderson, 2005).

Slightly more estimates fell on the same day (SD) as test in the 5-weeks condition $\left[F(2,57)=8.44, M S_{\mathrm{e}}=\right.$ $\left.1.07, p<.01, \eta_{\mathrm{p}}^{2}=.22\right]$, but the numbers of temporal error (TE) estimates were comparable across the conditions $\left[F(2,57)=1.55, M S_{\mathrm{e}}=2.93, p>.05, \eta_{\mathrm{p}}^{2}=.05\right]$.

\section{Analysis of Output Order}

A valid lag RRI was calculated for 900 responses by taking the RRI of a given response and subtracting it from the RRI of the immediately preceding response. These lag values ranged from -4 to +4 , where a lag of 0 represents the successive recall of events from the same RRI.

At any point in recall, it was possible for the participants to retrieve events from any one of the five different RRIs. Conditionalized response probabilities (CRPs) were calculated for every lag of each participant by dividing the number of observed transitions of each lag by the number of opportunities that the participant had to make such transitions. Figure 3 shows the lag CRP curves (Howard \& Kahana, 1999) for the three temporal conditions, showing that there was an increased tendency to recall successive events from the same RRI in all three temporal conditions (lag 0, CRP values ranging from .42 to .54).

An ANOVA was conducted on the three lags with the most data $(-1,0,+1)$. A 3 (temporal condition) $\times$ 3 (lag) mixed design ANOVA revealed a nonsignificant main effect of temporal condition $[F(2,55)<1]$, a significant main effect of lag $\left[F(2,110)=40.83, M S_{\mathrm{e}}=\right.$ $\left.0.03, p<.001, \eta_{\mathrm{p}}^{2}=.43\right]$, and a nonsignificant interac-

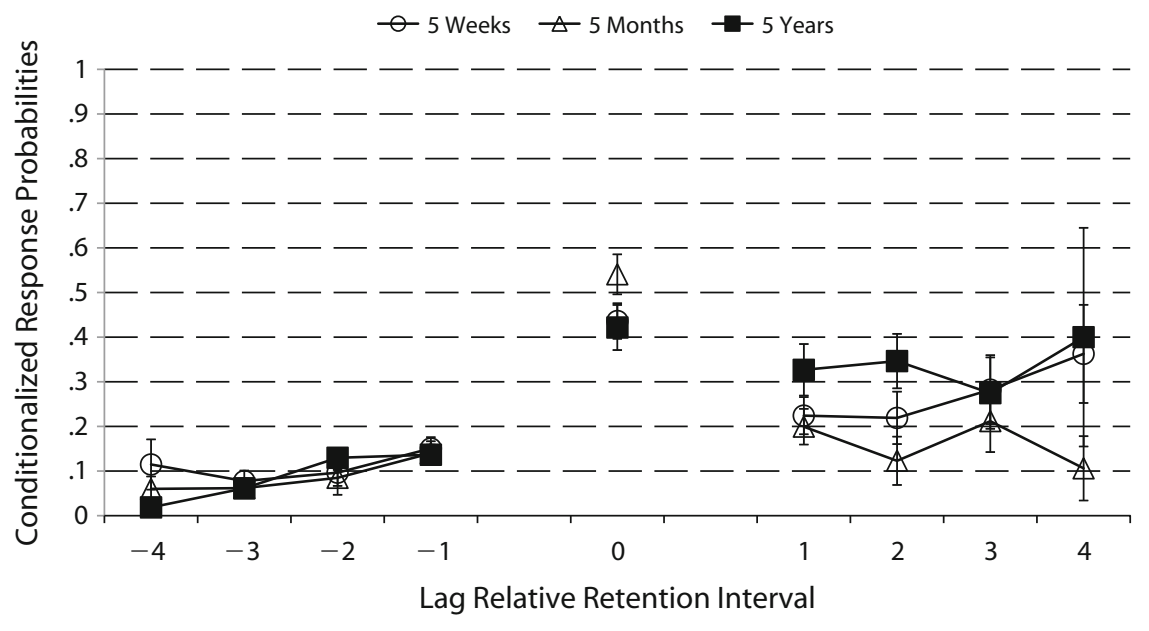

Figure 3. The lag conditionalized response probability (CRP) functions of recalled autobiographical events. $\mathrm{Lag}$ refers to the difference in relative retention intervals of successive responses, and the CRPs are calculated by dividing, for each participant, the different responses made at each lag by the number of opportunities that there were to make the different transitions. Error bars represent \pm 1 standard error. 
tion $\left[F(4,110)=2.28, M S_{\mathrm{e}}=0.03, p>.05, \eta_{\mathrm{p}}^{2}=.08\right]$. Pairwise comparisons revealed significantly higher CRPs for the lag 0 values.

We adapted a method developed by Howard et al. (2008) to control for a potential confound between contiguity effects and recency effects. All the pairs of RRIs that produced valid lag transitions were entered into a surrogate data set, these pairs were randomly shuffled 1,000 times, and the original lag transitions were calculated as a $z$ score with respect to this random distribution. The elevated CRPs at lag 0 were extremely robust, but once the effects of recency were controlled for, there was no further effect for $|\operatorname{lag}|$ values greater than 0 .

Finally, within the lag 0 transitions, the mean transitions were $1.53(\sigma=0.60), 6.49(\sigma=3.54)$, and $87.00(\sigma=$ $44.23)$ days for the 5 -weeks, 5 -months, and 5-years conditions, respectively, which, when analyzed as a proportion of the time scale, did not differ significantly $[F(2,56)<1]$.

\section{DISCUSSION}

Our findings are broadly consistent with time scale similarity in measures of retrieval rate, relative recency, and contiguity. Consistent with the results in Maylor et al. (2001), the participants retrieved the same number of autobiographical events from the three different time periods and did so at very similar rates, as predicted by time scale similarity in the retrieval rates from autobiographical memory.

The participants also produced highly similar relative retention functions in the three temporal conditions, findings broadly consistent with time scale similarity in relative recency effects, at least with the shortest and longest temporal periods. The SIMPLE model (Brown et al., 2007) provides a good account of the principle of relative recency, because, in SIMPLE, retrieval is a function of the relative temporal distinctiveness of the list items and the most recently stored items are thought to be the most distinct.

Finally, the participants exhibited a clear tendency across the temporal conditions to recall successive events from the same RRI. Consistent with the principle of temporal contiguity, similar temporal contiguity effects were observed over weeks, months, and years and were found at far greater time scales than had been reported previously (Howard et al., 2008; Unsworth, 2008).

Our data show that the items were not recalled from the same absolute retention functions, nor were there list length effects (Ward, 2002). One way to reconcile these differences is to acknowledge that there are important differences between autobiographical recall and recall of words from laboratory-generated lists.

First, there is the nature of the material to be remembered. In laboratory list-learning experiments, the tobe-recalled material shares the same unit (a word on the list) regardless of the list length. By contrast, in autobiographical recall, participants have the freedom to recall memories at different levels of specificity under different temporal conditions. Maylor et al. (2001) argued that pragmatic factors operate such that participants adopt a "natural" level of specificity appropriate to the time scale. For example, attending yesterday's biology lecture might be a more appropriate response for a participant retrieving from a short interval (e.g., last 5 weeks), rather than from a long temporal interval (e.g., last 5 years). Consistent with this claim, Moreton (2009) found that autobiographical events retrieved from longer time periods are rated by independent raters as being "more memorable" than those retrieved from shorter temporal periods.

Second, in laboratory list-learning experiments, the tobe-recalled items are randomly ordered. By contrast, there has been considerable theorizing concerning the organization of autobiographical memory (e.g., Brewer, 1986; Conway, 2005). For example, Conway claimed that episodic memories are organized within the autobiographical knowledge base into general events linked with particular lifetime periods within different themes. Thus, there are multiple ways in which autobiographical memories can be generated, such that our analysis by time necessarily confounds time with other semantic factors that shape retrieval.

These differences should not distract us from the interesting time scale similarities observed in our data. If one considers retrieval in this task in terms of temporal distinctiveness, Maylor et al. (2001; following Glenberg \& Swanson, 1986) argued that at longer time periods, there will be a greater number of to-be-remembered events of a given grain size but that these events will be harder to discriminate from their competitors. Thus, selecting events of larger "grain sizes" in the longer temporal conditions might help participants retrieve items that would otherwise be difficult to sample because of increased interitem competition. However, given that time may be confounded with other organizational factors in our data, other explanations of time scale similarity may be possible if participants use different time-dependent semantic cues to interrogate their autobiographical memories at different time scales.

We note that our retention functions are less steep than the power retention functions typically observed when participants are free to recall autobiographical memories from any time period (Galton, 1879; Rubin, 1982). However, when memories are cued within a specific time period, the retention function can change drastically. For example, Rubin and Baddeley (1989) asked researchers at the Applied Psychology Unit in Cambridge to name and date as many of the 76 speakers who had taken part in the colloquia series over a 2.5 -year period as they could. Their data showed a shallow forgetting function (relative to a power law). For modeling purposes, they preferred an Ebbinghaus-type retention function to describe the data, but because the data were not sufficient to distinguish among the various monotonically decreasing functions, they fit the data using linear regression.

Rubin and Baddeley (1989) also demonstrated that selfdating of autobiographical memories can be reasonably accurate. They compared participants' date estimates of the colloquia with the actual dates and found that although the mean absolute error increased with retention interval, the error tended to be free from systematic bias, being 
normally distributed around the actual date. Similarly, Moreton (2009) examined the recall of news events over different time scales. The retention functions obtained using self-dated estimates were very similar to those obtained using objective dating, giving us confidence that our retention functions were not a result of systematic bias in dating (for reviews of event dating, see Friedman, 1993, 2004; Huttenlocher, Hedges, \& Prohaska, 1988).

In some ways, our task resembles a semantic or verbal fluency task in which participants are free to recall any exemplar from a semantic category or any word starting with a given letter. One commonality in the data is the slowing of output (e.g., Bousfield \& Sedgewick, 1944; Gruenewald \& Lockhead, 1980). Since it seems unlikely that the participants were exhausting all their memories, we interpret the slowing as an example of output interference in recall (e.g., Laming, 2009; Roediger, 1978).

In summary, our findings are broadly consistent with accounts that predict time scale similarities in the retrieval of autobiographical events from differing time periods, at least in terms of retrieval rates, relative recency effects, and relative temporal contiguity effects.

\section{AUTHOR NOTE}

B.J.M. is now at the University of Nottingham. We thank Caren Rotello, David Rubin, Marc Howard, and an anonymous reviewer for their very helpful comments on an earlier version of the article. Correspondence concerning this article should be addressed to B. J. Moreton, University of Nottingham, Institute of Work, Health and Organisations, International House, Jubilee Campus, Wollaton Road, Nottingham NG8 1BB, England (e-mail: bryan.moreton@nottingham.ac.uk) or G. Ward, Department of Psychology, University of Essex, Wivenhoe Park, Colchester, Essex CO4 3SQ, England (e-mail: gdward@essex.ac.uk).

\section{REFERENCES}

Anderson, C. J. (2005). Calendar and reverse calendar effects: Time peaks in memory as a function of temporal cues. Memory, 13, 113 123. doi:10.1080/09658210344000620

Bousfield, W. A., \& Sedgewick, C. H. W. (1944). An analysis of sequences of restricted associative responses. Journal of General Psychology, 30, 149-165.

Brewer, W. F. (1986). What is autobiographical memory? In D. C. Rubin (Ed.), Autobiographical memory (pp. 25-49). Cambridge: Cambridge University Press.

Brown, G. D. A., Neath, I., \& Chater, N. (2007). A temporal ratio model of memory. Psychological Review, 114, 539-576. doi:10.1037/ 0033-295X.114.3.539

Conway, M. A. (2005). Memory and the self. Journal of Memory \& Language, 53, 594-628. doi:10.1016/j.jml.2005.08.005

Friedman, W. J. (1993). Memory for the time of past events. Psychological Bulletin, 113, 44-66. doi:10.1037/0033-2909.113.1.44
Friedman, W. J. (2004). Time in autobiographical memory. Social Cognition, 22, 591-605. doi:10.1521/soco.22.5.591.50766

Galton, F. (1879). Psychometric experiments. Brain, 2, 149-162. doi:10.1093/brain/2.2.149

Glenberg, A. M., \& Swanson, N. G. (1986). A temporal distinctiveness theory of recency and modality effects. Journal of Experimental Psychology: Learning, Memory, \& Cognition, 12, 3-15. doi:10.1037/ 0278-7393.12.1.3

Gruenewald, P. J., \& Lockhead, G. R. (1980). The free recall of category examples. Journal of Experimental Psychology: Human Learning \& Memory, 6, 225-240. doi:10.1037/0278-7393.6.3.225

Howard, M. W., \& KaHANA, M. J. (1999). Contextual variability and serial position effects in free recall. Journal of Experimental Psychology: Learning, Memory, \& Cognition, 25, 923-941. doi:10.1037/0278 $-7393.25 .4 .923$

HoWARD, M. W., \& KaHANA, M. J. (2002). A distributed representation of temporal context. Journal of Mathematical Psychology, 46, 269299. doi:10.1006/jmps.2001.1388

Howard, M. W., Youker, T. E., \& Venkatadass, V. S. (2008). The persistence of memory: Contiguity effects across hundreds of seconds. Psychonomic Bulletin \& Review, 15, 58-63. doi:10.3758/ PBR.15.1.58

Huttenlocher, J., Hedges, L., \& Prohaska, V. (1988). Hierarchical organization in ordered domains: Estimating the dates of events. Psychological Review, 95, 471-484. doi:10.1037/0033-295X.95.4.471

Laming, D. (2009). Failure to recall. Psychological Review, 116, $157-$ 186. doi:10.1037/a0014150

Maylor, E. A., Chater, N., \& Brown, G. D. A. (2001). Scale invariance in the retrieval of retrospective and prospective memories. Psychonomic Bulletin \& Review, 8, 162-167.

Moreton, B. J. (2009). Time scale similarity and long-term memory for autobiographical and news events. Unpublished doctoral thesis, University of Essex, Colchester, U.K.

MuRDOCK, B. B. (1962). The serial position effect of free recall. Journal of Experimental Psychology, 64, 482-488. doi:10.1037/h0045106

PenNingroth, S. (2005). Free recall of everyday retrospective and prospective memories: The intention-superiority effect is moderated by action versus state orientation and by gender. Memory, 13, 711-724. doi:10.1080/09658210444000359

Roediger, H. L., III (1978). Recall as a self-limiting process. Memory \& Cognition, 6, 54-63.

Rubin, D. C. (1982). On the retention function for autobiographical memory. Journal of Verbal Learning \& Verbal Behavior, 21, 21-38. doi:10.1016/S0022-5371(82)90423-6

Rubin, D. C., \& BadDeley, A. D. (1989). Telescoping is not time compression: A model of the dating of autobiographical events. Memory \& Cognition, 17, 653-661.

UNSWORTH, N. (2008). Exploring the retrieval dynamics of delayed and final free recall: Further evidence for temporal-contextual search. Journal of Memory \& Language, 59, 223-236. doi:10.1016/j.jml .2008 .04 .002

WARD, G. (2002). A recency-based account of the list length effect in free recall. Memory \& Cognition, 30, 885-892.

(Manuscript received November 26, 2009; revision accepted for publication February 1, 2010.) 